\title{
Reflectance Pulse Oximetry in Fetal Lambs
}

\author{
A. CARIN DASSEL, REINDERT GRAAFF, JAN G. AARNOUDSE, JAN M. ELSTRODT, \\ PIETER HEIDA, MARCO H. KOELINK, FRITS F. DE MUL, AND JAN GREVE
}

\begin{abstract}
Departments of Obstetrics and Gynecology, Center for Biomedical Technology, Central Animal Laboratory, Technical Research Department, University Hospital Groningen, Groningen [A.C.D., R.G., J.G.A., J.M.E., P.H.]; and Department of Applied Physics, University of Twente, Enschede [M.H.K., F.F.d.M., J.G.], The Netherlands
\end{abstract}

\begin{abstract}
Transmission pulse oximetry is used for monitoring in many clinical settings. However, for fetal monitoring during labor and in situations with poor peripheral perfusion, transmission pulse oximetry cannot be used. Therefore, we developed a reflectance pulse oximeter, which uses the relative intensity changes of the reflected red and infrared light (red/infrared ratio) to measure the arterial oxygen saturation. The performance of the reflectance pulse oximeter was studied in acute experiments in fetal lambs. By stepwise reduction of the inspired oxygen concentration of the ewe, measurements were done at the fetal scalp at various arterial oxygen saturation levels (17$82 \%$ ). Reflectance pulse oximeter readings were averaged over periods of $15 \mathrm{~s}$ and compared with simultaneously taken fetal arterial blood samples. A calibration curve for the relationship between red/infrared ratio and arterial oxygen saturation was obtained from 53 measurements in four fetal lambs, by linear regression analysis [red/infrared $\left.=4.088-\left(0.038 \cdot \mathrm{SaO}_{2}\right), r=0.96\right]$. In these experiments, the pulse oximeter showed a precision of $4.7 \%$ oxygen saturation around the calibration curve, with a $95 \%$ confidence interval of $\pm 9.4 \%$. (Pediatr Res 31: 266-269, 1992)
\end{abstract}

\section{Abbreviations}

R/IR, red/infrared

$\mathrm{SaO}_{2}$, arterial oxygen saturation

LED, light-emitting diode

$\mathrm{FiO}_{2}$, fraction of inspired oxygen

$\mathrm{PaO}_{2}$, arterial oxygen pressure

Pulse oximetry provides continuous, noninvasive monitoring of $\mathrm{SaO}_{2}(1,2)$. The method uses the pulsatile changes of red and infrared light transmitted through tissue to estimate arterial oxygenation. In adults, light transmission is usually measured through the finger, toe, ear, or nose. In newborns, light transmitted through the hand or foot is used for pulse oximetry. Limitations in accessible measurement sites due to circulatory problems and artefacts caused by motion impair the use of transmission pulse oximetry. With the fetal head as the presenting part, light transmission cannot be used for fetal pulse oximetry during labor. For this purpose a pulse oximeter that measures reflected light is required. This can be achieved by modifying commercially available transmission probes into reflectance probes, which have been used recently for studies in the human fetus during labor $(3,4)$.

In healthy adults, most pulse oximeters provide reliable and

Received June 4, 1991; accepted October 25, 1991.

Correspondence and reprint requests: Jan G. Aarnoudse, M.D., Ph.D., University Hospital Groningen, Depts. of Obstetrics \& Gynecology, Oostersingel 59, 9713 EZ Groningen, The Netherlands. accurate estimates of $\mathrm{SaO}_{2}$. However, it remains to be seen if pulse oximetry in the fetus is as accurate as in the adult. First, the influence of scattering is much more pronounced in the reflectance mode than it is in transmission (5-7). Second, the currently available pulse oximeters show a considerable decrease in accuracy at $\mathrm{SaO}_{2}$ below $70 \%$ (8), which is the predominant range in the fetus during labor. Finally, previous studies have shown that fetal scalp blood flow is markedly reduced in the course of labor (9-11). As a consequence, the pulse wave is likely to diminish, decreasing the signal to noise ratio, which causes a decrease in accuracy. For these reasons, we developed a reflectance pulse oximeter that has a well-defined geometry of the probe and displays directly the relative changes in R/IR ratio.

In the human fetus, it is practically impossible to compare $\mathrm{SaO}_{2}$ as estimated by pulse oximetry with values determined in arterial blood samples. Therefore, a study was undertaken in the ovine fetus to evaluate the performance of a reflectance pulse oximeter system developed in our department. Fetal $\mathrm{SaO}_{2}$ was measured over a wide range, from 17 to $82 \%$, and compared with the reflectance pulse oximetry readings.

\section{MATERIALS AND METHODS}

Animal preparation. Five ewes (mixed breed) with a gestational age of 124-134 d were used for this investigation. Guidelines for the care and use of animals approved by the local institution were followed. Food, but not water, was withheld for $24 \mathrm{~h}$ before operation. Anesthesia was induced by diazepam $2 \mathrm{mg} / \mathrm{kg}, 0.2$ $\mathrm{mg}$ glycopyrrolate, and ketamine $10 \mathrm{mg} / \mathrm{kg}$, i.v. The ewe was intubated and mechanically ventilated with an $\mathrm{N}_{2} \mathrm{O} / \mathrm{O}_{2}$ mixture, with positive end-expiratory pressure when necessary. Anesthesia was maintained with $0.5 \%$ halothane inhalation and ketamine infusion, $3 \mathrm{mg} / \mathrm{min}$. A catheter was placed in the maternal pedal artery for continuous measurement of blood pressure and for blood sampling. The intraabdominal temperature of the ewe was continuously measured and was kept near $39.5^{\circ} \mathrm{C}$ by means of a thermostatted heating blanket underneath the animal.

The ewe was placed in dorsal position, and the uterus was exposed by a paramedian abdominal incision. The fetal head and right forelimb were delivered through a small uterine incision. An arterial catheter was advanced via the right axillary artery into the thoracic aorta proximal to the ductus arteriosus; thereafter, the limb was replaced in utero. The probe of the reflectance pulse oximeter was applied onto the fetal scalp by means of a probe holder (Fig. 1), which was glued on the partly shaven scalp with cyanoacrylate. During the experiment, the fetal head was covered with a silver swaddle to prevent it from cooling.

Reflectance pulse oximeter. The reflectance pulse oximeter developed in our department determines $\mathrm{SaO}_{2}$ by comparing light intensity changes after reflectance in tissue. The ratio of the relative changes of the intensities at $660 \mathrm{~nm}$ (red) and $940 \mathrm{~nm}$ (infrared), $\mathrm{R} / \mathrm{IR}$, is used to determine the $\mathrm{SaO}_{2}$.

The probe contains two light sources (LED, Cerled types CR 10 SR and CR 10 IR; Electronic Consulting Services, Pfaffen- 


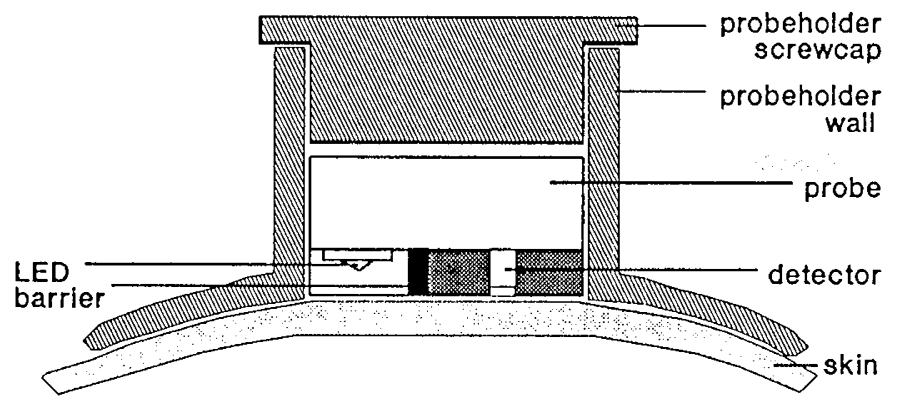

Fig. 1. A schematic drawing of the probe and the probeholder when applied on the skin.

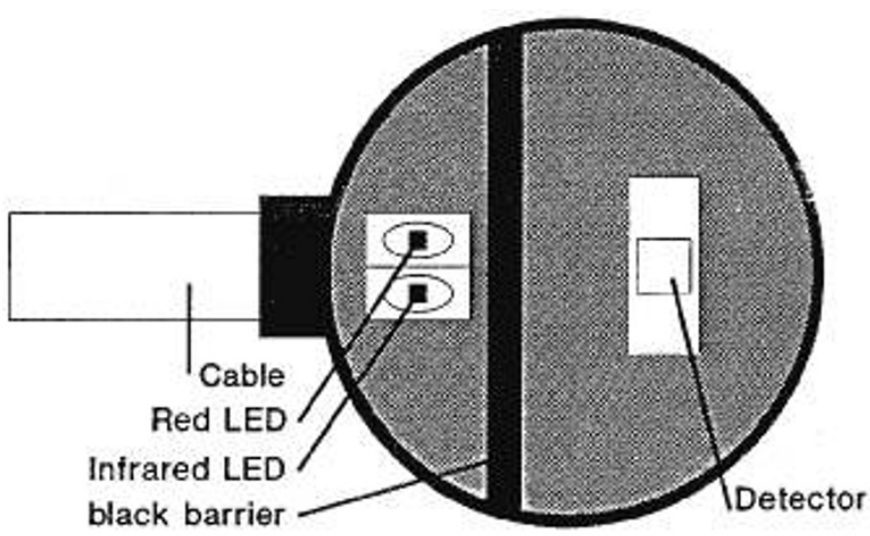

Fig. 2. Reflectance pulse oximeter probe: a schematic drawing.

hofen, Germany) on one side of an optical barrier and a detector, a photodiode (BPX 90, Siemens, München, Germany), on the opposite side of the optical barrier. Direct illumination of the photodiode by the LED (light shunting) is prevented by an optical barrier within the probe and by painting the inside of the probe and the surroundings of the detector black. Because the probe is applied in good contact with the skin by the probeholder, light shunting is also opposed. The distance between the LED and the photodiode is $7.5 \mathrm{~mm}$. A schematic drawing of the probe is shown in Figure 2.

Both LED illuminate the skin alternately during $20 \mu$ s pulses, with a repetition rate of $1 \mathrm{kHz}$. The signals are processed every $40 \mathrm{~ms}$ and converted to 12 -bit digital values. Further processing is performed with a personal computer. Red and infrared signals are recorded for a period of $15 \mathrm{~s}$. Each measurement over a 15$s$ epoch results in a mean $\mathrm{R} / \mathrm{IR}$ value with an $\mathrm{SD}$. The $\mathrm{SD}$ of the mean value reflects the quality of the measurement. If the SD exceeded $10 \%$ of the mean, measurements were not included.

Experimental procedures. Approximately $1 \mathrm{~h}$ after induction of anesthesia, measurements started. The $\mathrm{FiO}_{2}$ of the ewe $\left(\mathrm{O}_{2}\right.$ monitor IL 408; Instrumentation Laboratory Inc., Lexington, $\mathrm{MA})$ was elevated to $100 \%$ to obtain the higher fetal $\mathrm{SaO}_{2}$ levels. By stepwise reduction of $\mathrm{FiO}_{2}$, a steady state was achieved for 5 min at various levels of fetal $\mathrm{SaO}_{2}$. During these periods, the red and infrared signals were recorded for $15 \mathrm{~s}$ and, simultaneously, a fetal arterial blood sample was taken. When measurements at the lowest saturation levels were obtained, maternal $\mathrm{FiO}_{2}$ was again elevated to $100 \%$ to permit recovery of the animals. In each experiment, three to four runs were carried out.

The heparinized blood samples were introduced within $2 \mathrm{~min}$ into a blood-gas analyzer (ABL 330; Radiometer, Copenhagen, Denmark) for the measurement of $\mathrm{pH}, \mathrm{PO}_{2}$, and $\mathrm{PCO}_{2}$, corrected for the maternal body temperature. $\mathrm{SaO}_{2}$ was measured within 5 min, using a two-wavelength hemoximeter (OSM2; Radiometer) calibrated for blood of lambs, assuming only oxyhemoglobin and deoxyhemoglobin to be present. $\mathrm{SaO}_{2}$ was measured in duplicate, and the average value is reported. A small number of blood samples could not be analyzed immediately; these were stored on ice and analyzed within half an hour.

Statistics. The R/IR values from the reflectance pulse oximeter were related to the measured $\mathrm{SaO}_{2}$ by means of linear regression analysis, which gave the estimated slope and the intercept of the regression line. The regression line was used as a calibration curve to transform $\mathrm{R} / \mathrm{IR}$ values to saturation values in a linear way (predicted $\mathrm{SaO}_{2}$ ). By comparing two saturation values, the predicted value and the measured $\mathrm{SaO}_{2}$, it is possible to calculate the precision.

To compare two methods, Altman $(12,13)$ recommends calculation of the mean and the SD of the difference. The mean of the difference is called "bias" or "accuracy." It shows a systemic overestimation or underestimation of one method relative to the other. The bias calculated for the comparison of the reflectance pulse oximeter saturation with the arterial blood samples has no value in our study, inasmuch as we used the same data to obtain the calibration curve. The SD of the difference is often referred to as "precision" and will represent the "variability" or "random error" (2). The precision is meaningful in our study because it describes the random error of the instrument.

\section{RESULTS}

In one of the five sheep studied, mechanical ventilation caused large artefacts in the pulse oximetry recordings; therefore, the R/ IR values of this animal could not be used.

From the remaining four fetal lambs, 58 measurements were obtained. Five measurements were excluded because the SD of the mean $\mathrm{R} / \mathrm{IR}$ ratio over the 15 -s measurement period exceeded $10 \%$ of the mean. Thus, 53 data pairs were left for comparison. $\mathrm{SaO}_{2}$ was varied over a wide range, from 17 to $82 \%$. For the pooled data of all experiments, the relationship between pulse oximeter readings (R/IR ratio) and $\mathrm{SaO}_{2}$ of the arterial blood samples was determined by linear regression analysis (Fig. 3). The equation for the best fitted linear regression line was R/IR $=4.088-\left(0.038 \cdot \mathrm{SaO}_{2}\right)(\%)$. The regression analysis revealed a high degree of correlation $(r=0.96)$ and a standard error of the $y$ estimate of 0.18 .

To make comparison with other pulse oximeter studies possible, the R/IR values were transformed to predicted $\mathrm{SaO}_{2}$ values. With the regression line, predicted oxygen saturation values of the reflectance pulse oximeter were calculated (Fig. 4). The pulse oximeter showed a precision of $4.7 \%$ oxygen saturation around the calibration curve, with a $95 \%$ confidence interval of $\pm 9.4 \%$.

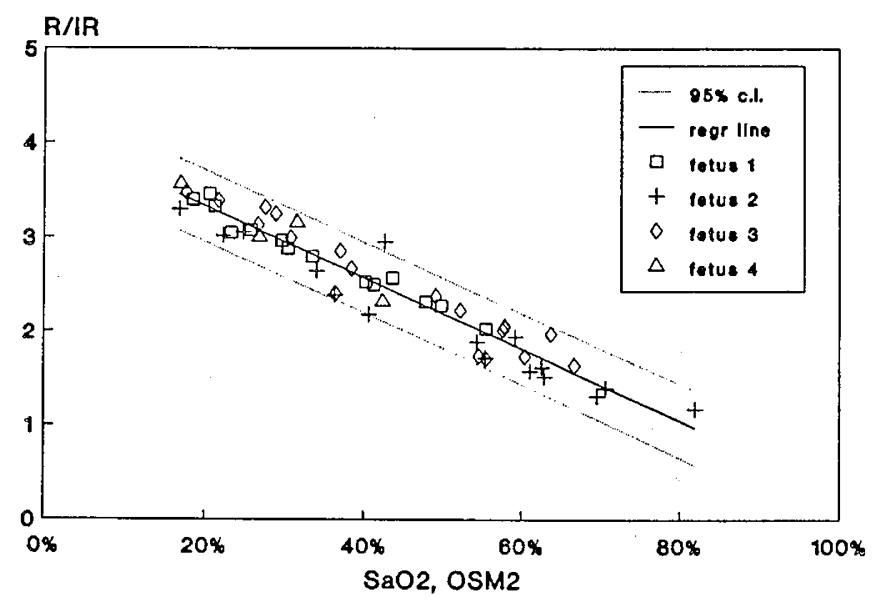

Fig. 3. Comparison of $\mathrm{SaO}_{2}$ of blood samples (OSM2) ( $x$ axis) and the $\mathrm{R} / \mathrm{IR}$ ratios measured by the reflectance pulse oximeter ( $y$ axis) in four fetal lambs. $y=4.088-0.0388 x, r=0.96$, standard error of the estimate $=0.179 ; n=53$. The solid line represents the linear regression line, whereas the dotted lines represent the $95 \%$ confidence interval (c.i.). 


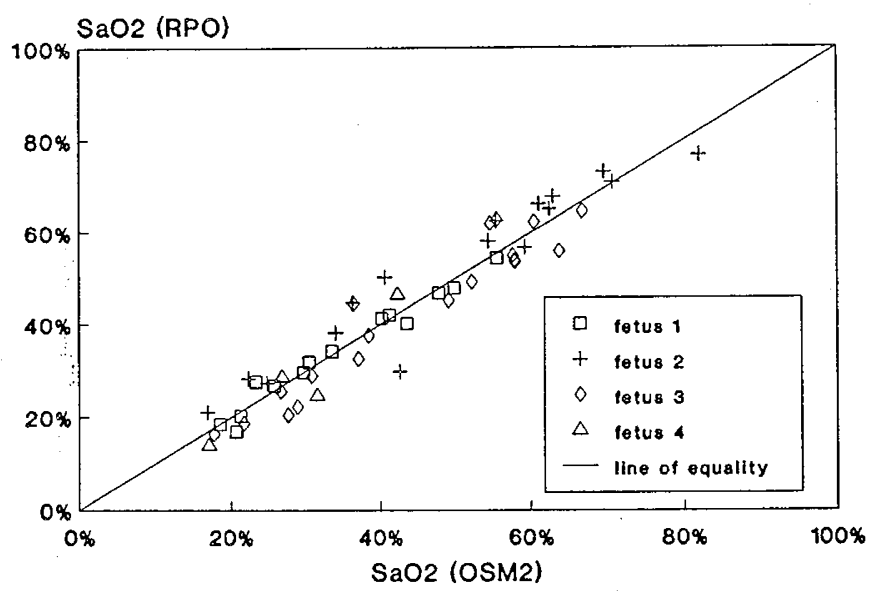

Fig. 4. Oxygen saturation values derived from measurements with the reflectance pulse oximeter ( $R P O$ ) at the fetal scalp ( $y$ axis) vs $\mathrm{SaO}_{2}$ from blood samples (OSM2) ( $x$ axis).

\section{DISCUSSION}

Monitoring of fetal oxygenation by continuous measurement of transcutaneous oxygen tensions $\left(\mathrm{PaO}_{2}\right)$ during labor has severe limitations (14). Changes in blood oxygen concentration, over the steep portion of the fetal oxygen dissociation curve, are more precisely reflected by $\mathrm{SaO}_{2}$ than by $\mathrm{PaO}_{2}$ (15). Therefore, measuring $\mathrm{SaO}_{2}$ instead of $\mathrm{PaO}_{2}$ has several advantages. In the human fetus, pulse oximetry has been attempted by using a transmission pulse oximeter with an adapted probe for reflectance measurements at the fetal scalp $(3,4,11)$, but comparison with $\mathrm{SaO}_{2}$ measured in arterial blood samples was not possible in those studies. Therefore, we decided to investigate the performance of reflection pulse oximetry in the fetal lamb.

The fetal sheep provides a good model for pulse oximetry studies because light absorption by $\mathrm{Hb}$ in the range of spectra used in pulse oximetry is hardly different among mammals. The scatter characteristics of sheep erythrocytes, however, may differ from human erythrocytes because of dissimilarity in size and shape. Skin properties of fetal sheep also will be different from those of human fetuses. The results, therefore, might not be directly applicable to the human situation.

The influence of scattering is much more pronounced in reflectance pulse oximetry than in transmission pulse oximetry (5-7). As a consequence, commercially available transmission pulse oximeters with adapted probes for reflectance measurements are expected to estimate $\mathrm{SaO}_{2}$ less accurately. Moreover, these pulse oximeters are not calibrated for in vivo measurements below $\mathrm{SaO}_{2}$ measurements $<50 \%$. Therefore, we developed a reflectance pulse oximeter that has a well-defined geometry of light sources and detector in the probe and displays directly the relative changes in $\mathrm{R} / \mathrm{IR}$ ratio.

In the present study, pulse oximeter values were omitted when, during a 15 -s measurement period, the SD differed more than $10 \%$ from the mean value. This occurred in five of the 58 pulse oximetry measurements. Theoretically, this might be explained by real variability of $\mathrm{SaO}_{2}$. However, a more detailed analysis of the signals indicates that this is unlikely. The relatively high SD in these cases appeared mainly when signal strength was low and when slow blood volume fluctuations synchronous with maternal ventilation were present. The results of an entire experiment were unsuitable because of such ventilation effects. Because a pulse oximeter analyzes any pulsatile absorber, it is obvious that fluctuations in venous filling disturb pulse oximetry measurements (16-18).

In the remaining cases, a weak pulse wave causing an extremely low signal to noise ratio seemed responsible for the increased variability. As the light intensity fluctuations caused by the pulse amplitude become smaller, electronic and physiologic noises become more significant. Most commercially available transmission pulse oximeters stop readings or give the sign "low-quality signal" when the fluctuating part of the infrared pulse size becomes less than $0.2 \%$ of the detected light intensity (19). In the present study, signals are obtained from measurements with a low pulse size (mean $0.14 \%$, range $0.05-0.35 \%$ ), with $79 \%$ of the data having a pulse size below $0.2 \%$.

A decrease in scalp perfusion because of vasoconstriction caused by the hypoxic stress or resulting from cooling of the skin could well explain the occasional poor pulse wave recordings. It is not yet known if the considerable reduction in scalp blood flow that usually occurs in the human fetus during the course of labor will permit reliable pulse oximetry monitoring (9-11).

Figure 3 shows that linear regression adequately describes the relation between the $\mathrm{SaO}_{2}$ and the $\mathrm{R} / \mathrm{IR}$ ratio of the present data measured with our reflectance pulse oximeter. Mendelson $e t$ al. (20) also used linear regression to find the relation between these parameters in reflectance pulse oximetry. For transmission pulse oximeters, however, Wukitsch et al. (19) applied a nonlinear relationship between the $\mathrm{R} / \mathrm{IR}$ ratio and $\mathrm{SaO}_{2}$ measurements.

The precision of our measurements was $4.7 \% \mathrm{SaO}_{2}$. This is not as good as the reported values of commercially available transmission pulse oximeters at higher oxygen saturations (70-100\%), which are 1.5 to $3 \%$ in adults (19). For oxygen saturations $<50 \%$, the precision is not reported; most pulse oximeters do not even display a saturation value. Experimental studies have demonstrated that when $\mathrm{SaO}_{2}$ becomes less than $60 \%$, pulse oximetry becomes less accurate $(21,22)$. This is partly explained by the calibration curves in the pulse oximeters used being derived from studies in healthy adult volunteers and extrapolated to the lower oxygen saturation range. The reliability of pulse oximetry at low levels of oxygen saturation is essential if the technique will be of value for fetal monitoring (23).

The results of these experimental studies indicate that reflectance pulse oximeter measurements at the fetal scalp show a precision that is promising, even at low $\mathrm{SaO}_{2}$ levels, despite low pulse amplitudes. Improved data processing is likely to result in more accurate measurements of fetal $\mathrm{SaO}_{2}$.

Acknowledgment. The authors thank Professor W. G. Zijlstra, M.D., Ph.D., for reviewing the manuscript.

\section{REFERENCES}

1. Kelleher FK 1989 Pulse oximetry. J Clin Monit 5:37-62

2. Tremper KK, Barker SJ 1989 Pulse oximetry. Anesthesiology 70:98-109

3. Johnson N, Johnson VA, Bannister J, Lyons G, Lilford RJ, Griffiths-Jones M Tuffnell D, Onwude JL 1990 Monitoring the fetus with a pulse oximeter during a caesarean section. Br J Obstet Gynaecol 97:653-658

4. Peat S, Booker M, Lanigan C, Ponte J 1988 Continuous intrapartum measurement of fetal oxygen saturation. Lancet 2:213

5. Neuman MR 1987 Pulse oximetry: physical principles, technical realization and present limitations. Adv Exp Med Biol 220:135-143

6. Mendelson Y, Ochs BD 1988 Noninvasive pulse oximetry utilizing skin reflectance photoplethysmography. IEEE Trans Biomed Eng 35:798-805

7. Graaff R, Dassel ACM, Aarnoudse JG, Zijlstra WG 1991 Biophysical aspects of reflection pulse oximetry. In: Lafeber H, Aarnoudse JG, Jongsma H (eds) Fetal and Neonatal Physiological Measurements. Elsevier, Amsterdam, pp 129-134

8. Sendak MJ, Harris AP, Donham RT 1988 Accuracy of pulse oximetry during arterial oxyhemoglobin desaturation in dogs. Anesthesiology 68:111-114

9. Smits TM, Aarnoudse JG 1984 Variability of fetal scalp blood flow during labour: continuous transcutaneous measurement by the laser Doppler technique. Br J Obstet Gynaecol 91:524-531

10. Smits TM, Aarnoudse JG, Zijlstra WG 1990 Scalp blood flow measured by laser Doppler flowmetry and transcutaneous $\mathrm{PO}_{2}$ and $\mathrm{PCO}_{2}$ in the lamb. Pediatr Res 27:442-446

11. Johnson N, Johnson VA, Bannister J, Lilford RJ 1990 The effect of caput succedaneum on oxygen saturation measurements. Br J Obstet Gynaecol 97:493-498

12. Altman DG 1980 Statistics and ethics in medical research V: analyzing data Br Med J 281:1473-1475

13. Bland JM, Altman DG 1986 Statistical methods for assessing agreement between two methods of clinical measurement. Lancet 1:307-310 
14. Smits TM, Aarnoudse JG, Zijlstra WG 1989 Fetal scalp blood flow as recorded by laser Doppler flowmetry and transcutaneous $\mathrm{PO}_{2}$ during labour. Early Hum Dev 20:109-124

15. Fanconi S 1988 Reliability of pulse oximetry in hypoxic infants. J Pediatr 112: 424-427

16. Yelderman M 1990 Pulse oximetry. In: CD Blitt (ed) Monitoring in Anesthesia and Critical Care Medicine. Churchill Livingstone, London, pp 417-427

17. Kim JM, Arakawa K, Benson KT, Fox DK 1986 Pulse oximetry and circulatory kinetics associated with pulse volume amplitude measured by photoelectric plethysmography. Anesth Analg 65:1333-1339

18. Scheller JS, Loeb R 1988 Respiratory artifact during pulse oximetry in critically ill patients. Anesthesiology 69:602-603
19. Wukitsch MW, Petterson MT, Tobler DR, Pologe JA 1988 Pulse oximetry: analysis of theory, technology, and practice. J Clin Monit 4:290-301

20. Mendelson Y, Kent JC, Yocum BL, Birle MJ 1988 Design and evaluation of a new reflectance pulse oximeter sensor. Biomed Instrum Technol 22: $167-173$

21. Severinghaus JW, Naifeh KH 1987 Accuracy of response of six pulse oximeters to profound hypoxia. Anesthesiology 67:551-558

22. Severinghaus JW, Naifeh $\mathrm{KH}$, Koh SO 1989 Errors in 14 pulse oximeters during profound hypoxia. J Clin Monit 5:72-81

23. Huch R, Huch A, König V, Neuman MR, Parker D, Yount J, Lübbers D 1988 Limitations of pulse oximetry. Lancet 1:357-358

\section{Announcement}

\section{First International Workshop "Iodine Deficiency in Europe: A Continuing Concern"}

The First International Workshop "Iodine Deficiency in Europe: A Continuing Concern" will be held in Brussels, Belgium, April 24-28, 1992. Topics will include thyroid growth in iodine deficiency and excess; epidemiologic approaches to the evaluation of iodine nutrition in developed countries; an update of the iodine nutrition status in European countries, including the former "Eastern block"; consequences of iodine deficiency in pregnant women and their offspring; comparison of Europe and iodine-replete areas; iodine deficiency and nuclear hazards; and prophylaxis of iodine deficiency in Europe: action at the governmental level. For information, contact the Secretariat: F. Delange, Department of Radioisotopes, Hospital Saint-Pierre, 322, eu Haute, 1000Brussels, Belgium, phone: 3225354563 or 53545 64, FAX: 3225354006. 